\title{
Gall-inducing insects from Serra do Cabral, Minas Gerais, Brazil
}

\author{
Marcel Serra Coelho ${ }^{1,3}$, Marco Antônio Alves Carneiro' \\ Cristina Alves Branco ${ }^{I}$ \& Geraldo Wilson Fernandes ${ }^{I}$ \\ ${ }^{1}$ Laboratório de Ecologia Evolutiva e Biodiversidade, Departamento de Biologia Geral - DBG, \\ Instituto de Ciências Biológicas - ICB, Universidade Federal de Minas Gerais - UFMG, CP 486, \\ CEP 30161-970 Belo Horizonte, MG, Brazil \\ ${ }^{2}$ Laboratório de Entomologia Ecológica, Departamento de Biodiversidade, Evolução e Meio \\ Ambiente - DEBIO, Instituto de Ciências Exatas e Biológicas - ICEB, \\ Universidade Federal de Ouro Preto - UFOP, Campus Morro do Cruzeiro, \\ CEP 35400-000, Ouro Preto, MG, Brasil \\ ${ }^{3}$ Corresponding author: Marcel Serra Coelho, e-mail: marcel.s.coelho@gmail.com
}

COELHO, M.S., CARNEIRO, M.A.A., BRANCO, C. \& FERNANDES, G.W. Gall-inducing insects from Serra do Cabral, Minas Gerais, Brazil. Biota Neotrop. 13(3): http://www.biotaneotropica.org.br/v13n3/en/ abstract?article+bn02113032013

Abstract: Our goal was describe the gall richness through the characterization of their external shapes and occurrence patterns in their host plants from Parque Estadual da Serra do Cabral. In a universe of 34 families, 64 genera and 89 plant species, 47 gall-inducing insects in 21 families, 32 genera and 39 host plant species were recorded. The families, which hosted higher gall richness, were those with highest abundance. Asteraceae represented $33 \%$ of the species collected followed by Malpighiaceae $8 \%$ and Fabaceae $8 \%$, each one concentrating $25 \%, 19 \%$ and $8 \%$ of gall-inducing insects, respectively. The organ most attacked was the leaf (51\%), followed by the stem (42\%) and the terminal branch (4\%). Ninety-six percent (96\%) of galls were glabrous. Only $25.5 \%$ of the galls described in our study have been already recorded in previous studies, reinforcing the need to increase the sampling effort toward a better understanding of the richness, distribution, and natural history of gall-inducing insects from Brazil.

Keywords: biodiversity, biogeography, herbivore, host plant, insect-plant interaction.

COELHO, M.S., CARNEIRO, M.A.A., BRANCO, C. \& FERNANDES, G.W. Insetos indutores de galhas da Serra do Cabral, Minas Gerais, Brasil. Biota Neotrop. 13(3): http://www.biotaneotropica.org.br/v13n3/pt/ abstract?article+bn02113032013

Resumo: Objetivo deste estudo foi descrever a riqueza de morfotipos de galha por meio da caracterização de suas formas e os padrões de ocorrência em suas plantas hospedeiras no Parque Estadual da Serra do Cabral. Num universo de 34 famílias, 64 gêneros e 89 espécies de plantas, foram registradas 47 espécies de galhas induzidas por insetos em 21 famílias, 32 gêneros e 39 espécies de plantas hospedeiras. As famílias que concentraram maior riqueza de insetos galhadores foram as famílias mais abundantes. A família Asteraceae representou 33\% das espécies coletadas, seguida das Malpighiaceae 8\% e Fabaceae 8\%, cada uma concentrando 25\%, 19\% e 8\% das espécies de insetos galhadores, respectivamente. O órgão mais atacado foi a folha (51\%), seguido do caule (42\%) e ramo terminal (4\%). Noventa e seis por cento (96\%) das galhas foram glabras. O fato de apenas $25 \%$ das galhas descritas neste estudo já terem sido registradas em trabalhos prévios, reforça a necessidade em aumentar o esforço amostral na direção de um maior conhecimento sobre a riqueza, distribuição e história natural dos insetos indutores de galhas no Brasil.

Palavras-chave: biodiversidade, biogeografia, herbivoria, planta hospedeira, interação inseto-planta. 


\section{Introduction}

Galls are anomalous structures from portions of organs or plant tissues that develop in response to the presence of an inducer organism, often an insect (Price 2005, Shorthouse et al. 2005). Gall development is the result of the interaction between the insect inductor and the host plant, where the insect acquires control over the host plant, diverting its resources away from growth, development and defense (Abrahamson \& Weis 1997, Stone \& Schönrogge 2003, Fernandes et al. 2010).

Gall-inducing insects and their host plants have been widely studied in recent decades in different Brazilian physiognomies, e.g.: Pantanal (Wetlands) (Julião et al. 2002), Amazon (Julião et al. 2005), Restinga (Brazilian Coastal Vegetation) (Maia 2001, 2005, Maia et al. 2002, Mendonça 2007, Oliveira \& Maia; 2005), Atlantic Forest (Fernandes et al. 2001, Fernandes \& Negreiros 2006, Santos et al. 2012), Cerrado (Brazilian Savannah) (Fernandes et al. 1988, Maia \& Fernandes 2004, Gonçalves-Alvim \& Fernandes 2001), Rupestrian Fields (Carneiro et al. 2009b), Tropical Dry Forest on Limestone Outcrops (Coelho et al. 2009) and Caatinga (Tropical Dry Forest) (Santos et al. 2011a). Despite this effort, some ecosystems were only recently sampled, such as the Tropical Dry Forest on Limestone Outcrops (Coelho et al. 2009), Altitude Wetland Forests (Santos et al. 2011b), Caatinga (Santos et al. 2011a) and Amazon (Almada \& Fernandes 2011, Maia 2012).

The cerrado has the more galling surveys among Brazilian ecosystems, but due its large territory, there are many gaps to be filled to its local biodiversity. This work is part of a project with the goal of describing the natural history of gall-inducing insects, their galls, and their host plants from the Brazilian Cerrado and, in particular, from Rupestrian Fields. In a recent study, Carneiro et al. (2009b) recorded the gall richness in six distinct regions across the Espinhaço range, Minas Gerais. In this work, galls from Parque Estadual da Serra do Cabral (PESC) were described and characterized by their external morphology and their host plant occurrence.

\section{Material and Methods}

Samples were collected in two physiognomies of Cerrado: open fields (Campo Cerrado) and shrubby fields (Campo Sujo) in Parque Estadual da Serra do Cabral (PESC), located between the coordinates $17^{\circ} 03^{\prime} \mathrm{S}-18^{\circ} 13^{\prime} \mathrm{S}$ and $44^{\circ} 05^{\prime} \mathrm{W}-44^{\circ} 52^{\prime} \mathrm{W}$. An area of 250,000 ha, with an altitudinal ranging from $600 \mathrm{~m}$ to $1385 \mathrm{~m}$, in the Minas Gerais state, Brazil. The PESC climate is classified as Aw (Köppen classification), with two well defined seasons, hot and rainy summers, and cold and dry winters; an annual rainfall average of $750 \mathrm{~mm}$ and an average temperature of $22^{\circ} \mathrm{C}$. Samples were collected in April, 2008, the end of the rainy season. As part of the Espinhaço Range, PESC presents typical Cerrado physiognomies, rupestrian fields, gallery forests and Altitude Fields (Hatschbach et al. 2006).

Sampling was carried out according to standard methodology used to study galls diversity in Rupestrian Fields and Cerrado (Fernandes \& Price 1988, Carneiro et al. 2009b). Along the altitudinal gradient, 10 points were arbitrarily defined ranging from $879 \mathrm{~m}$ to $1.255 \mathrm{~m}$, with an altitude range of $376 \mathrm{~m}$. The samples at higher altitudes were conducted in areas covered by Rupestrian Fields; at intermediate and low altitudes, samples were conducted on areas covered by Cerrado, Rupestrian Fields and Open Fields (Campo Cerrado). Forests, areas close to trails and any areas with visible human interference were excluded from the sampling.

Gall sampling was performed following the methodology described by Fernandes \& Price (1988, but see Price et al. 1998). At each sampling point, a plot with 100 woody plants of shrub stature (between 0.3 and $2 \mathrm{~m}$ high) was arbitrarily selected, totaling 1,000 plants across the mountain. Each plant was sampled throughout the aerial part of the individual by counting directly the number of galls. According to Carneiro et al. (2009a) gall description associated with the identification of the host-plant species is a reliable indication of the galling insect richness. About $95 \%$ of described species of Cecidomyiidae from Brazil can be identified based on their external shape associated with the host plant on which they occur, reinforcing the use of this methodology as reliable in galling studies (Price et al. 1998, Blanche 2000, Cuevas-Reyes et al. 2003, 2004, Oyama et al. 2003). The sampled host plants and their galls were mounted and deposited in the herbariums OUPR e BHCB (acronyms according to Holmgren et al. 1990). The collected plants were separated into families and were then identified by specialists to the lowest taxonomic level possible. The classification of plant species followed the system proposed by Angiosperm Phylogeny Group III (2009). The galls were photographed and characterized according to the color, shape, presence or absence of hairs, and plant organ where they occur (see Carneiro et al. 2009b). Galling insects taxa were always identified when possible.

\section{Results}

In PESC 47 gall species within 21 families, 32 genera and 39 species of host plants have been found. In total, 34 families, 64 genera and 89 species of plants were sampled (Table 1, 2, Figure 1-3). The most abundant families hosted the highest gall richness. Asteraceae represented $33 \%$ of the species collected, followed by Malpighiaceae $8 \%$ and Fabaceae $8 \%$, each one concentrating $25 \%, 19 \%$ and $8 \%$ of gall species, respectively. The genera that concentrated the highest richness of galls were Byrsonima (Malpighiaceae) with 13\% and Lessingianthus (Asteraceae) with $8 \%$. The species with the greatest galls richness was Byrsonima guilleminiana A.Juss. with 3 galls $(6 \%)$. The genera and species that concentrated the most gall richness belong to the plant families with the highest occurrence, Asteraceae (33\%), Fabaceae (8\%) and Malpighiaceae (29\%). The Cecidomyiidae (Diptera) family was the most frequent ( $93 \%$ ), followed by Coleoptera (4\%) and Hymenoptera (2\%). The most common gall shapes were discoid (15\%), fusiform (23\%), globulous (23\%), intumescence $(10 \%)$, rolled edge $(10 \%)$, elliptical $(6 \%)$, terminal branch $(4 \%)$ conical (6\%) and rolled (2\%). The organ most attacked was the leaf (51\%), followed by the stem (42\%) and the terminal branch (4\%). Ninety-six percent $(96 \%)$ of galls were glabrous.

\section{Discussion}

In this study, we found 47 of galling insect species, and only $12(25.5 \%)$ had been reported in previous studies. Previous studies have reported Duguetia furfuracea [Table 1, Figure 1b, UrsoGuimarães et al. 2003, Urso-Guimarães \& Scareli-Santos 2006, Malves \& Frieiro-Costa 2012, Saito \& Urso-Guimarães 2012], Aspidosperma tomentosum [Table 1, Figure 1c, Gonçalves-Alvim \& Fernandes 2001, Araújo et al. 2011)], Baccharis salzmannii [Table 1, Figure 1f, Carneiro et al. 2009b], Eremanthus erythropappus [Table 1, Figure 1h, Carneiro et al. 2009b, Saito \& Urso-Guimarães 2012], Lessingianthus tomentellus [Table 1, Figure 1m, Carneiro et al. 2009b], Jacaranda paucifoliata [Table 1, Figure 1p, Carneiro et al. 2009b], Tabebuia ochracea (Cham.) Standl. [Table 1, Figure 1q, UrsoGuimarães et al. 2003], Kielmeyera coriacea [Table 1, Figure 2a, Carneiro et al. 2009b], Microlicia confertiflora [Table 1, Figure 3a, Carneiro et al. 2009b], Eugenia punicifolia [Table 1, Figure 3b, Carneiro et al. 2009b, Saito \& Urso-Guimarães 2012], Palicourea rigida [Table 1, Figure 3e], and Vochysia elliptica [Table 1, Figure 3g, Carneiro et al. 2009b], all with one gall mosphotype. The fact that only $23 \%$ of the galls described in this study had been recorded in 
Coelho, M.S. et al.

Table 1. Host plants, description of galls at a Cerrado from Serra do Cabral, Minas Gerais, Brazil.

\begin{tabular}{|c|c|c|c|c|c|c|c|}
\hline Host Plants & $\begin{array}{l}\text { Likely gall maker } \\
\text { taxa }\end{array}$ & Organ & Shape & Color & Pubescence & Chambers & Photos \\
\hline \multicolumn{8}{|l|}{ Annonaceae } \\
\hline \multirow[t]{2}{*}{ Duguetia furfuracea (A. St.-Hil.) Saff. } & Cecidomyiidae & leaf & elliptical & green & glabrous & 1 & 1(a) \\
\hline & Cecidomyiidae & leaf & globulous & green & glabrous & 1 & 1(b) \\
\hline \multicolumn{8}{|l|}{ Apocynaceae } \\
\hline Aspidosperma tomentosum Mart. & Cecidomyiidae & leaf & discoid & green & glabrous & 1 & $1(\mathrm{c})$ \\
\hline \multicolumn{8}{|l|}{ Asteraceae } \\
\hline Acritopappus longifolius (Gardner) R.M. King \& H. Rob. & Cecidomyiidae & stem & rolled edge & green & glabrous & 1 & $1(\mathrm{~d})$ \\
\hline Aspilia jolyana G. M. Barroso & Cecidomyiidae & leaf & rolled edge & green & glabrous & 1 & $1(\mathrm{e})$ \\
\hline \multirow[t]{2}{*}{ Baccharis salzmannii DC. } & Cecidomyiidae & stem & fusiform & brown & glabrous & 1 & $1(\mathrm{f})$ \\
\hline & Cecidomyiidae & leaf & elliptical & green & glabrous & 1 & $1(\mathrm{~g})$ \\
\hline \multirow[t]{2}{*}{ Eremanthus erythropappus (DC.) MacLeish } & Asphondylia serrata & leaf & globulous & brown & glabrous & 1 & 1(h) \\
\hline & Cecidomyiidae & stem & fusiform & brown & glabrous & 1 & 1(i) \\
\hline \multirow[t]{2}{*}{ Lessingianthus coriaceus (Less.) H. Rob. } & Cecidomyiidae & leaf & globulous & green & glabrous & 1 & $1(\mathrm{j})$ \\
\hline & Cecidomyiidae & stem & intumescence & brown & glabrous & various & $1(\mathrm{k})$ \\
\hline Lessingianthus hoveaefolius (Gardner) H. Rob. & Cecidomyiidae & leaf & globulous & green & glabrous & 1 & $1(1)$ \\
\hline Lessingianthus tomentellus (Mart. ex DC.) H. Rob. & Cecidomyiidae & stem & terminal branch & brown & glabrous & various & $1(\mathrm{~m})$ \\
\hline Lychnophoriopsis heterotheca Sch. Bip. & Cecidomyiidae & stem & globulous & brown & glabrous & 1 & $1(\mathrm{n})$ \\
\hline Piptocarpha rotundifolia (Less.) Baker & Cecidomyiidae & leaf & discoid & green & glabrous & 1 & $1(0)$ \\
\hline \multicolumn{8}{|l|}{ Bignoniaceae } \\
\hline Jacaranda paucifoliata Mart. ex DC. & Cecidomyiidae & stem & rolled & green & glabrous & 1 & $1(\mathrm{p})$ \\
\hline Tabebuia ochracea (Cham.) Standl. & Cecidomyiidae & leaf & intumescence & brown & hairy & 1 & $1(\mathrm{q})$ \\
\hline \multicolumn{8}{|l|}{ Bixaceae } \\
\hline Cochlospermum regium (Schrank) Pilg. & Cecidomyiidae & leaf & conical & green & glabrous & 1 & $1(\mathrm{r})$ \\
\hline \multicolumn{8}{|l|}{ Chrysobalanaceae } \\
\hline Licania humilis Cham. \& Schltdl. & Cecidomyiidae & leaf & discoid & brown & glabrous & 1 & $1(\mathrm{~s})$ \\
\hline Licania nitida Hook. f. & Cecidomyiidae & & rolled edge & green & glabrous & 1 & $1(\mathrm{t})$ \\
\hline \multicolumn{8}{|l|}{ Clusiaceae } \\
\hline Kielmeyera coriacea Mart. \& Zucc. & Cecidomyiidae & leaf & discoid & brown & glabrous & 1 & 2(a) \\
\hline \multicolumn{8}{|l|}{ Convolvulaceae } \\
\hline Merremia tomentosa Hallier & Cecidomyiidae & leaf & rolled edge & green & glabrous & 1 & 2(b) \\
\hline \multicolumn{8}{|l|}{ Erythroxylaceae } \\
\hline Erythroxylum campestre A. St.-Hil. & Cecidomyiidae & stem & globulous & brown & glabrous & 1 & 2(c) \\
\hline \multicolumn{8}{|l|}{ Euphorbiaceae } \\
\hline Maprounea guianensis Aubl. & unidentified & leaf & rolled edge & green & glabrous & various & 2(d) \\
\hline \multicolumn{8}{|l|}{ Fabaceae } \\
\hline Calliandra asplenioides (Nees) Benth. ex Jackson & Cecidomyiidae & stem & fusiform & brown & glabrous & 1 & 2(e) \\
\hline Chamaecrista geminata (Benth.) H.S. Irwin \& Barneby & Cecidomyiidae & stem & intumescence & brown & glabrous & 1 & 2(f) \\
\hline Machaerium opacum Vogel & Cecidomyiidae & stem & fusiform & brown & glabrous & 1 & $2(\mathrm{~g})$ \\
\hline Mimosa polycarpa Kunth & Cecidomyiidae & leaf & globulous & green & hairy & 1 & 2(h) \\
\hline \multicolumn{8}{|l|}{ Lamiaceae } \\
\hline Hyptis eriophylla Pohl ex Benth. & Cecidomyiidae & stem & fusiform & green & glabrous & 1 & 2(i) \\
\hline \multicolumn{8}{|l|}{ Lauraceae } \\
\hline Ocotea lancifolia (Schott) Mez & Cecidomyiidae & leaf & globulous & green & glabrous & 1 & 2(j) \\
\hline \multicolumn{8}{|l|}{ Lythraceae } \\
\hline Diplusodon uninervius Koehne & Cecidomyiidae & stem & fusiform & brown & glabrous & 1 & $2(\mathrm{k})$ \\
\hline \multicolumn{8}{|l|}{ Malpighiaceae } \\
\hline Banisteriopsis campestris (A. Juss.) Little & Cecidomyiidae & leaf & discoid & green & glabrous & 1 & 2(1) \\
\hline
\end{tabular}


Table 1. Continued..

\begin{tabular}{|c|c|c|c|c|c|c|c|}
\hline Host Plants & $\begin{array}{c}\text { Likely gall maker } \\
\text { taxa }\end{array}$ & Organ & Shape & Color & Pubescence & Chambers & Photos \\
\hline \multirow[t]{2}{*}{ Banisteriopsis laevifolia (A. Juss.) B. Gates } & Cecidomyiidae & stem & globulous & brown & glabrous & 1 & $2(\mathrm{~m})$ \\
\hline & Coleoptera & stem & fusiform & brown & glabrous & 1 & $2(\mathrm{n})$ \\
\hline Byrsonima crassa Nied. & Cecidomyiidae & leaf & discoid & green & glabrous & 1 & $2(0)$ \\
\hline \multirow[t]{3}{*}{ Byrsonima guilleminiana A. Juss. } & Cecidomyiidae & leaf & discoid & green & glabrous & 1 & $2(\mathrm{p})$ \\
\hline & Cecidomyiidae & leaf & elliptical & brown & glabrous & 1 & 2(q) \\
\hline & Cecidomyiidae & stem & intumescence & brown & glabrous & 1 & $2(\mathrm{r})$ \\
\hline Byrsonima pachyphylla A. Juss. & Coleoptera & stem & intumescence & brown & glabrous & 1 & $2(\mathrm{~s})$ \\
\hline Byrsonima sp. & Cecidomyiidae & leaf & discoid & green & glabrous & 1 & $2(\mathrm{t})$ \\
\hline \multicolumn{8}{|l|}{ Melastomataceae } \\
\hline Microlicia confertiflora DC. & Cecidomyiidae & stem & fusiform & brown & glabrous & 1 & 3(a) \\
\hline \multicolumn{8}{|l|}{ Myrtaceae } \\
\hline Eugenia punicifolia (Kunth) DC. & Hymenoptera & stem & fusiform & brown & glabrous & 1 & $3(b)$ \\
\hline \multicolumn{8}{|l|}{ Nyctaginaceae } \\
\hline \multirow[t]{2}{*}{ Guapira noxia (Netto) Lundell } & Cecidomyiidae & stem & globulous & brown & glabrous & various & $3(\mathrm{c})$ \\
\hline & Cecidomyiidae & leaf & discoid & green & glabrous & 1 & $3(d)$ \\
\hline \multicolumn{8}{|l|}{ Rubiaceae } \\
\hline Palicourea rigida Kunth & Cecidomyiidae & leaf & discoid & green & glabrous & 1 & $3(\mathrm{e})$ \\
\hline \multicolumn{8}{|l|}{ Verbenaceae } \\
\hline Lippia microphylla Cham. & Cecidomyiidae & $\begin{array}{l}\text { terminal } \\
\text { branch }\end{array}$ & intumescence & brown & glabrous & & $3(\mathrm{f})$ \\
\hline Vochysiaceae & & & & & & 1 & \\
\hline Vochysia elliptica Mart. & Cecidomyiidae & stem & fusiform & brown & glabrous & 1 & $3(\mathrm{~g})$ \\
\hline
\end{tabular}

Table 2. Number of gall-inducing insects associated with its plant families at a Cerrado from Serra do Cabral, MG. Families without galls were listed as "other families".

\begin{tabular}{lccccc}
\hline \multirow{2}{*}{ Families } & \multicolumn{2}{c}{ Plants } & & \multicolumn{2}{c}{ Galls } \\
\cline { 2 - 3 } \cline { 5 - 6 } Annonaceae & 1 & 1.3 & & Richness & \% \\
\hline Apocynaceae & 1 & 1.3 & & 1 & 4.3 \\
Asteraceae & 26 & 33.3 & & 12 & 25.1 \\
Bignoniaceae & 2 & 2.6 & & 2 & 4.3 \\
Bixaceae & 1 & 1.3 & & 1 & 2.1 \\
Chrysobalanaceae & 2 & 2.6 & & 2 & 4.3 \\
Clusiaceae & 1 & 1.3 & & 1 & 2.1 \\
Convolvulaceae & 1 & 1.3 & & 2.1 \\
Erythroxylacee & 1 & 1.3 & & 1 & 2.1 \\
Euphorbiaceae & 3 & 3.8 & & 1 & 2.1 \\
Fabaceae & 6 & 7.7 & & 4 & 8.5 \\
Lamiaceae & 2 & 2.6 & & 2.1 \\
Lauraceae & 1 & 1.3 & & 2.1 \\
Lythraceae & 1 & 1.3 & & 2.1 \\
Malpighiaceae & 6 & 7.7 & 9 & 19.1 \\
Melastomataceae & 3 & 3.8 & 1 & 2.1 \\
Myrtaceae & 3 & 3.8 & 1 & 2.1 \\
Nyctaginaceae & 1 & 1.3 & 2 & 4.3 \\
Rubiaceae & 1 & 1.3 & 1 & 2.1 \\
Verbenaceae & 2 & 2.6 & 1 & 2.1 \\
Vochysiaceae & 1 & 1.3 & 1 & 2.1 \\
Outras famín & 12 & 15.4 & 0 & 0.0 \\
Total & 78 & 100 & 47 & 100 \\
\hline & & & &
\end{tabular}

previous studies reinforces the need to increase the sampling efforts of gall-inducing insects in the Espinhaço Range.

Using the same methods Carneiro et al. (2009b) recorded higher gall-inducing insects richness at different regions along the Espinhaço Range $(\mathrm{PE}$ Rio Preto $=75, \mathrm{RPPN}$ Caraça $=71, \mathrm{PE} \mathrm{Biribiri}=63, \mathrm{PE}$ Itacolomi $=59$, PE Serra do Ouro Branco $=50$ ) than PE Serra do Cabral (=47), except for PE Grão Mogol (=18). Thus, the PESC is the area with the second lowest richness of gall-inducing insects in the Espinhaço Range. This fact can be partly explained by the absence of super hosts, species that concentrate a large number of gall-inducing insects (sensu Veldtman \& McGeoch 2003). The regions with lower gall-inducing insect richness from the Espinhaço Range, PESC and PE Grão Mogol (Carneiro et al. 2009b) are also areas where species of the genus Baccharis were represented by only one host plant species [B. platypoda, (PESC) or where they were absent (PE Grão Mogol)]. Baccharis is an important genus that concentrates much of the galling insect richness of the Rupestrian Fields (Carneiro et al. 2009b).

The families Asteraceae, Fabaceae, Melastomataceae, Malpighiaceae and Myrtaceae are the most frequent in different Brazilian Cerrado physiognomies (Giulietti et al. 1987, Giulietti \& Pirani 1988) as well as in PESC (Hatschbach et al. 2006). These families alone concentrated $52 \%$ of the gall insect richness from PESC. Some studies report greater gall-inducing insect richness in families and genera richest in host species (Fernandes 1992, Blanche $\&$ Westody 1995). Studies in other Brazilian ecosystems have shown similar patterns, such as Cerrado (Gonçalves-Alvim \& Fernandes 2001), Rupestrian Fields (Maia \& Fernandes 2004, Carneiro et al. 2009b), Atlantic Forests (Fernandes et al. 2001), Tropical Dry Forests (Coelho et al. 2009), Seasonal Sub-tropical Forest (Mendonça 2007). 


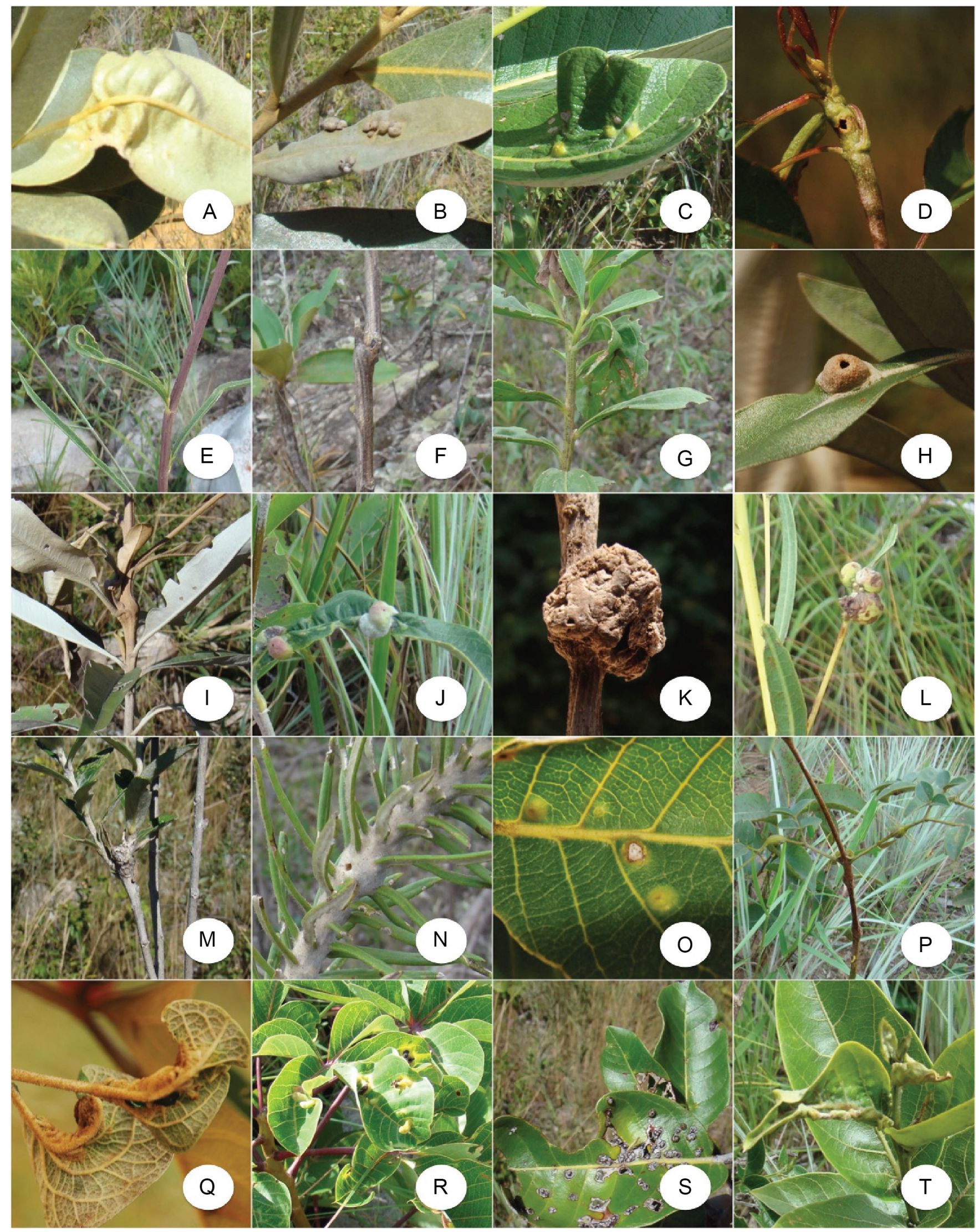

Figure 1. Host plants and its galls at a Cerrado from Serra do Cabral, Minas Gerais, Brazil. Annonaceae [Duguetia furfuracea (a-b)], Apocynaceae [Aspidosperma tomentosum (c)], Asteraceae [Acritopappus longifolius (d), Aspilia jolyana (e), Baccharis salzmanii (f-g), Eremanthus erythropappus (h-i), Lessingianthus coriaceus (j-k), Lessingianthus hoveaefolius (1), Lessingianthus tomentellus $(\mathrm{m})$, Lychnophoriopsis heterotheca $(\mathrm{n})$, Piptocarpha rotundifolia $(\mathrm{o})]$, Bignoniaceae [Jacaranda paucifoliata (p), Tabebuia ochracea (q)], Bixaceae [Cochlospermum regium (r)], Chrysobalanaceae [Licania humilis (s), Licania nitida (t)]. 


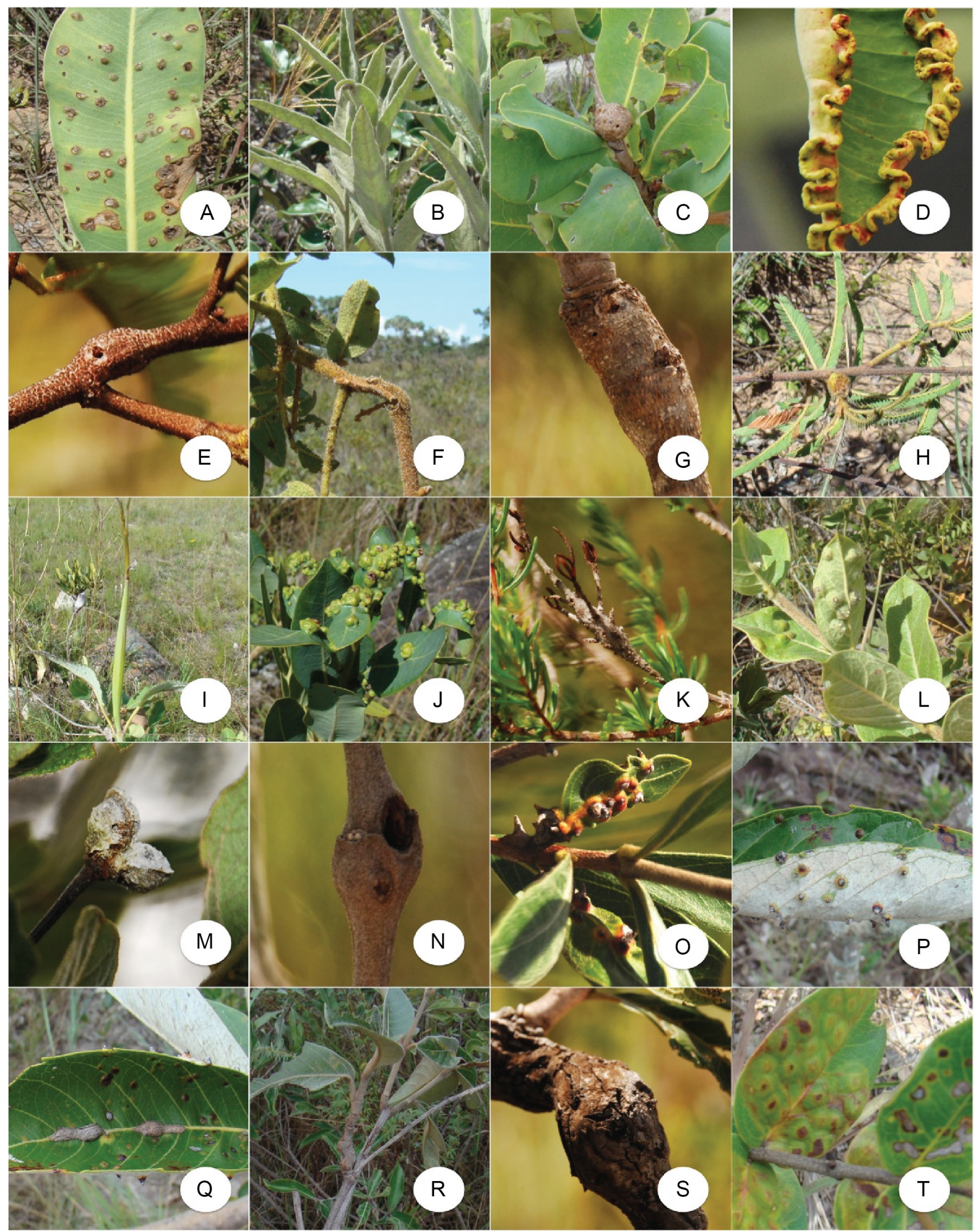

Figure 2. Host plants and its galls at a Cerrado from Serra do Cabral, Minas Gerais, Brazil. Clusiaceae [Kielmeyera coriacea (a)], Convolvulaceae [Merremia tomentosa (b)], Erythroxylaceae [Merremia tomentosa (c)], Euphorbiaceae [Maprounea guianensis (d)], Fabaceae [Calliandra asplenioides (e), Chamaecrista geminata (f), Machaerium opacum (g), Mimosa polycarpa (h)], Lamiaceae [Hyptis eriophylla (i)], Lauraceae [Ocotea lancifolia (j)], Lythraceae [Diplusodon uninervius $(\mathrm{k})$ ], Malpighiaceae [Banisteriopsis campestris (1), Banisteriopsis laevifolia $(\mathrm{m}, \mathrm{n})$, Byrsonima crassa $(\mathrm{o})$, Byrsonima guilleminiana $(\mathrm{p}, \mathrm{q}, \mathrm{r})$, Byrsonima pachyphylla (s), Byrsonima sp. (t)]. 


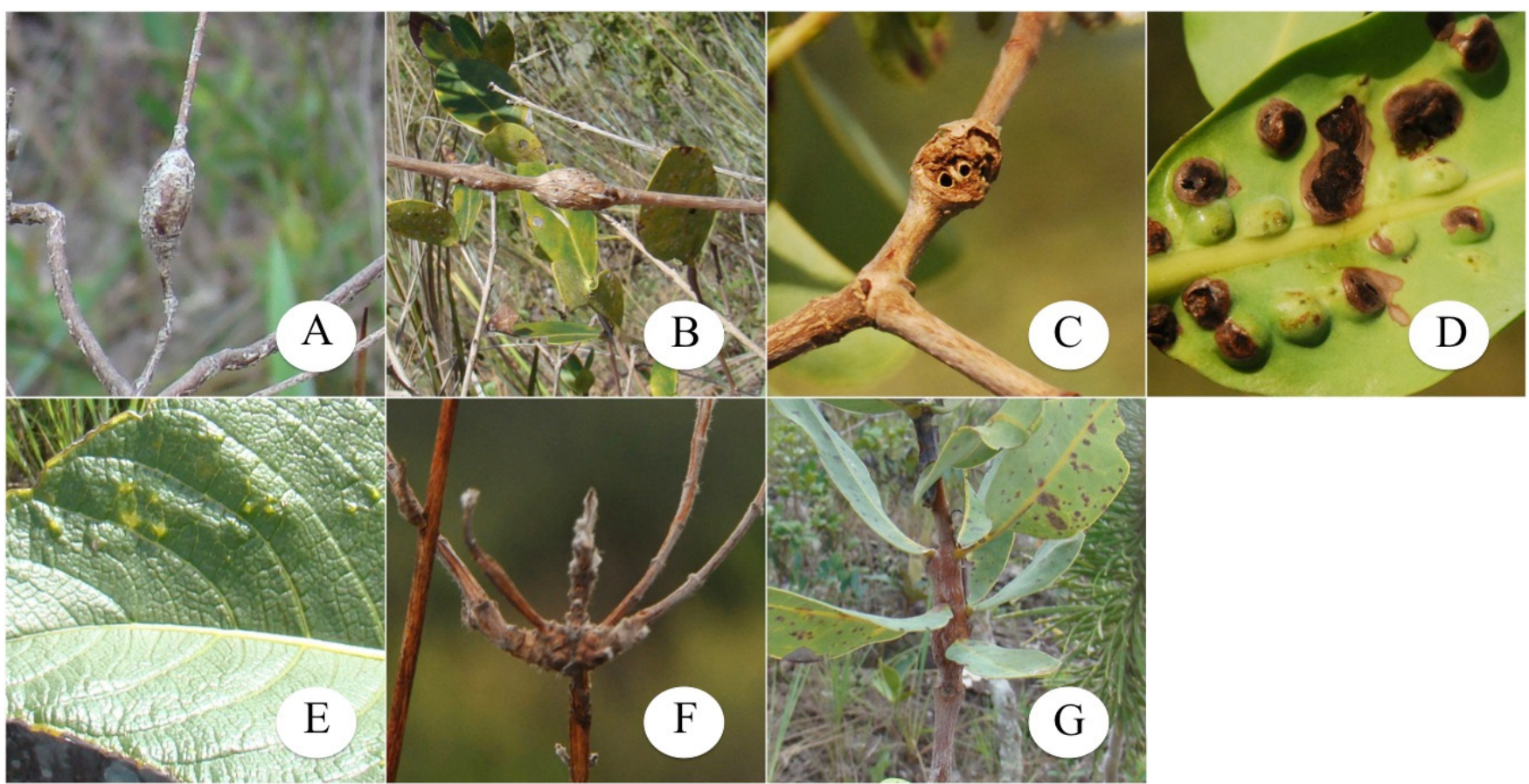

Figure 3. Host plants and its galls at a Cerrado from Serra do Cabral, Minas Gerais, Brazil. Melastomataceae [Microlicia confertiflora (a)], Mystaceae [Eugenia punicifolia (b)], Nyctaginaceae [Guapira noxia (c-d)], Rubiaceae [Palicourea rigida (e)], Verbenaceae [Lippia microphylla (f)], Vochysiaceae [Vochysia elliptica $(\mathrm{g})]$.

In this study, $93 \%$ of galling species belong to the Cecidomyiidae (Diptera) family, reflecting the great species richness of this family in Brazil, and in the Neotropics (Gagné 1994, Fernandes et al. 2001, Julião et al. 2002, Cuevas-Reyes et al. 2004, Maia 2005). As in other studies conducted in different biomes, such as Cerrado (Maia \& Fernandes 2004), Atlantic Rain Forest (Fernandes \& Negreiros 2006), Pantanal (Julião et al. 2002), Tropical Dry Forests (Coelho et al. $2009), 51 \%$ of galling insects occurred on leaves.

Studies relating to richness patterns and to the natural history of gall-inducing insects in Brazil are still incipient (Maia 2005). A study on global richness of gall-inducing insects estimated the existence of 21,000 to 211,000 species (Espírito-Santo \& Fernandes 2007). This inaccuracy is probably due to the lack of more studies thoughout the many ecosystems around the globe. Every new study focusing on gall-inducing insects inventories reports to science at least a $50 \%$ of new species (see Coelho et al. 2009). Therefore, further studies are needed in order to achieve a better understanding of the gall-inducing insect distribution in different Brazilian ecosystems.

\section{Acknowledgments}

We thank the two anonymous reviewers for the critical review and suggestions on the manuscript, Valeri Garcia for the English improvement and Dr. João Renato Stehmann (UFMG) for plant identifications. The Instituto Estadual de Florestas I.E.F. for logistical support. To FAPEMIG (2893/98) and CNPq (472811/2006-1; 30.9633 / 2007-9) for financial support. To CAPES for the scholarship awarded to M. S. Coelho and M. A. A. Carneiro.

\section{References}

ABRAHAMSON, W.G. \& WEIS, A.E. 1997. Evolutionary ecology across three trophic levels: goldenrods, gallmakers and natural enemies. Princeton University Press, New Jersey, 456p.
ALMADA, E.D. \& FERNANDES, G.W. 2011. Insetos indutores de galhas em florestas de terra- firme em reflorestamentos com espécies nativas na Amazônia Oriental. Bol. Mus. Para. Emilio Goeldi. Ser. Cienc. Nat. $6(2): 1-10$.

ANGIOSPERM PHYLOGENY GROUP - APG. 2009. An update of the Angiosperm Phylogeny Group classification for the orders and families of flowering plants: APG III. Bot. J. Linnean Soc. 161(2):105-121. http:// dx.doi.org/10.1111/j.1095-8339.2009.00996.x

ARAÚJO, W.S., SANTOS, B.B. \& GOMES-KLEIN, V.L. 2011. Insect galls from Serra dos Pirineus, GO, Brazil. Biota Neotrop. 11(2):357-365. http:// dx.doi.org/10.1590/S1676-06032011000200034

BLANCHE, K.R. 2000. Diversity of insect-induced galls along a temperaturerainfall gradient in the tropical savannah region of the Northern Territory, Australia. Austral Ecol. 25(4):311-318. http://dx.doi.org/10.1046/j.14429993.2000.01040.x

BLANCHE, K.R. \& WESTOBY, M. 1995. Gall-forming insect diversity is linked to soil fertility via host plant taxon. Ecology 76(7):2334-2337. http://dx.doi.org/10.2307/1941706

CARNEIRO, M.A.A., BRANCO, C.S.A., BRAGA, C.E.D., ALMADA, E.D., COSTA, M.B.M., FERNANDES, G.W. \& MAIA, V.C. 2009a. Are gall midge species (Diptera: Cecidomyiidae) host plant specialists? Rev. bras. Entomol. 53(3):365-378. http://dx.doi.org/10.1590/S008556262009000300010

CARNEIRO, M.A.A., BORGES, R.A.X., ARAÚJO, A.P.A. \& FERNANDES, G.W. 2009b. Insetos indutores de galhas da porção sul da Cadeia do Espinhaço, MG. Rev. Bras. Entomol. 53(4):570-592. http://dx.doi. org/10.1590/S0085-56262009000400007

COELHO, M.S., ALMADA, E.D., FERNANDES, G.W., CARNEIRO, M.A.A., SANTOS, R.M., QUINTINO, A.V. \& SANCHEZ-AZOFEIFA, A. 2009. Gall inducing arthropods from a seasonally dry tropical forest in Serra do Cipó, Brazil. Rev. Bras. Entomol. 53(3):404-414. http://dx.doi. org/10.1590/S0085-56262009000300015

CUEVAS-REYES, P., SIEBE, C., MARTÍNEZ-RAMOS, M. \& OYAMA, K. 2003. Species richness of gall-forming insects in a tropical rain forest: correlations with plant diversity and soil fertility. Biodivers. Conserv. 12(3):411-422. http://dx.doi.org/10.1023/A:1022415907109 
CUEVAS-REYES, P., QUESADA, M., HANSON, P., DIRZO, R. \& OYAMA, K. 2004. Diversity of gall-inducing insects in a Mexican tropical dry Forest: the importance of plant species richness, life forms, host plant age and plant density. J. Trop. Ecol. 92(4):707-716. http://dx.doi. org/10.1111/j.0022-0477.2004.00896.x

ESPÍRITO-SANTO, M.M. \& FERNANDES, G.W. 2007. How many species of gall-inducing insects are there on earth, and where are they? Ann. Entomol. Soc. Am. 100(2):95-99.

FERNANDES, G.W. 1992. Plant age and size effects on insular gall-forming species richness. GLOBAL. Ecol. Biogeogr. 2(3):71-74. http://dx.doi. org $/ 10.2307 / 2997508$

FERNANDES, G.W., COELHO, MS. \& LÜTTGE, U. 2010. Photosynthetic efficiency of Clusia arrudae leaf tissue with and without Cecidomyiidae galls. Braz. J. Biol. 70(3):723-728. PMid:21085778. http://dx.doi. org/10.1590/S1519-69842010000400004

FERNANDES, G.W., JULIÃO, G.R., ARAÚJO, R.C., ARAÚJO, S.C., LOMBARDI, J.A., NEGREIROS, D. \& CARNEIRO, M.A.A. 2001 Distribution and morphology of insect galls of the Rio Doce Valley, Brazil. Naturalia. 26(5):211-244.

FERNANDES, G.W. \& NEGREIROS, D. 2006. A comunidade de insetos galhadores da RPPN Fazenda Bulcão, Aimorés, Minas Gerais, Brasil. Lundiana. 7(2):111-120.

FERNANDES, G.W. \& PRICE, P.W. 1988. Biogeographical gradients in galling species richness: tests of hypotheses. Oecologia. 76:161-167. http://dx.doi.org/10.1007/BF00379948

FERNANDES, G.W., EUGÊNIO, T.N. \& MARTINS R.P. 1988. Ocorrência e caracterização de galhas entomógenas na vegetação do campus pampulha da universidade federal de Minas Gerais. Rev. Bras. Entomol. 5(1):11-29.

GAGNÉ, R.J. 1994. The gall midges of the region neotropical. Comstock: Ithaca.

GIULIETTI, A.M., MENEZES, N.L., PIRANI, J.R., MEGURO, M.L. \& WANDERLEY, M.G.L. 1987. Flora da Serra do Cipó: Caracterização e lista de espécies. Bol. Bot. Univ. S. Paulo 9(1):1-152.

GIULIETTI, A.M. \& PIRANI, J.R. 1988. Patterns of Geographic distribution of some Plant Species from the Espinhaço Range, Minas Gerais, Brazil. In Proceedings of a Workshop on Neotropical Biodiversity Distribution Patterns (P.E. Vanzolini \& W.R. Heyer, ed.) Academia Brasileira de Ciências, Rio de Janeiro, p.39-69.

GONÇALVES-ALVIM, S.J. \& FERNANDES, G.W. 2001. Biodiversity of galling insects: historical, community and habitat effects in four neotropical savannas. Biodivers. Conserv. 10(1):79-98. http://dx.doi. org/10.1023/A:1016602213305

HATSCHBACH, G., GUARÇONI E.A.E., SARTORI, M.A. \& RIBAS, O.S. 2006. Apectos fisionômicos da vegetação da Serra do Cabral - Minas Gerais - Brasil. Bol. Mus. Bot. Mun. 67:1-22.

HOLMGREN, P.K., HOLMGREN, N.H. \& BARNETT, L.C. 1990. Index Herbariorum. Part I: The Herbaria of The World. New York Botanical Garden, New York.

JULIÃO, G.R., AMARAL, M.E.C. \& FERNANDES, G.W. 2002. Galhas de insetos e suas plantas hospedeiras no Pantanal sul-mato-grossense. Naturalia. 27:47-74.

JULIÃO, G.R., VENTICINQUE, E.M., FERNANDES, G.W. \& KRAUS, J.E. 2005. Richness and abundance of gall-forming insects in the Mamirauá Varzea, a flooded Amazoniam forest. Uakari. 1(1):39-42.

MALVES, K. \& FRIEIRO-COSTA, A. 2012. List of Plants with galls induced by insects from the UNILAVRAS/Boqueirão Biological Reserve, Ingaí, state of Minas Gerais, Brazil. CheckList 8(3):426-431.

MAIA, V.C. 2001. The gall midges (Diptera, Cecidomyiidae) from three restingas of Rio de Janeiro State, Brazil. Rev. Bras. Zool. 18(2):583-629. http://dx.doi.org/10.1590/S0101-81752001000200028

MAIA, V.C. 2005. Catálogo dos Cecidomyiidae (Diptera) do estado do Rio de Janeiro. Biota Neotrop $<$ bibEm $>$. </bibEm>5(2):1-26. http://dx.doi. org/10.1590/S1676-06032005000300014
MAIA, V.C. 2012. Characterization of insect galls, gall makers, and associated fauna of Platô Bacaba (Porto de Trombetas, Pará, Brazil). Biota Neotrop. 11(4):1-17.

MAIA, V.C. \& FERNANDES, G.W. 2004. Insect galls from Serra de São José (Tiradentes, MG, Brazil.). Rev. Bras. Biol. 6(3):423-445.

MAIA, V.C., AZEVEDO, M.A.P. \& COURI, M.S. 2002. New contribution to the knowledge of the gall midges (Diptera, Cecidomyiidae) from the restinga of Barra de Maricá (Rio de Janeiro, Brazil). Stud Diptero. 9(2):447-452.

MENDONÇA, M.S. 2007. Plant diversity and galling arthropod diversity searching for taxonomic patterns in an animal-plant interaction in the neotropics. Bol. Soc. Arg. Bot. 42(3):347-357.

OYAMA, K., PÉREZ-PÉREZ, M.A., CUEVAS-REYES, P. \& LUNA-REYES, R. 2003. Regional and local species richness of gall-inducing insects in two tropical rain forests in Mexico. J. Trop. Ecol. 19(5):595-598. http:// dx.doi.org/10.1017/S0266467403003651

OLIVEIRA, J.C. \& MAIA, V.C. 2005. Ocorrência e caracterização de galhas de insetos na restinga de Grumari (Rio de Janeiro, RJ, Brasil). Arq. Mus. Nac. 63(4):669-675.

PRICE, P.W. 2005. Adaptative radiation of gall-inducing insects. Basic Appl. Ecol. 6(5):413-421. http://dx.doi.org/10.1016/j.baae.2005.07.002

PRICE, P.W., FERNANDES, G.W., LARA, A.C.F., BRAWN, J., GERLING, D., BARRIOS, H., WRIGHT, M.G., RIBEIRO, S.P. \& ROTHCLIFF, N. 1998. Global patterns in local number of insect galling species. J. Biogeogr. 25(3):581-591. http://dx.doi.org/10.1046/j.1365-2699.1998.2530581.x

SAITO, V.S. \& URSO-GUIMARÃES, M.V. 2012. Characterization of galls, insect galls and associated fauna of Ecological Station of Jataí (Luiz Antônio, SP). Biota Neotrop. 12(3):99-107. http://dx.doi.org/10.1590/ S1676-06032012000300011

SANTOS, J.C., ALMEIDA-CORTEZ, J.S. \& FERNANDES, G.W. 2011a. Righness of gall-inducing insects in the tropical dry forest (caatinga) of Pernambuco. Rev. Bras. Entomol. 55(1):45-54. http://dx.doi.org/10.1590/ S0085-56262011000100009

SANTOS, J.C.; ALMEIDA-CORTEZ, J.S. \& FERNANDES, G.W. 2011 b. Diversity of gall- inducing insects in the hight altitude wetland forests in Pernambuco, Northeastern Brazil. Braz. J. Biol. 71(1):47-56. PMid:21437398. http://dx.doi.org/10.1590/S1519-69842011000100008

SANTOS, J.C.; ALMEIDA-CORTEZ, J.S. \& FERNANDES, G.W. 2012. Gall-inducing insects from Atlantic forest of Pernambuco, Northeastern Brazil. Biota Neotrop. 12(3):197-213. http://dx.doi.org/10.1590/S167606032012000300020

SHORTHOUSE, J.D., WOOL, D. \& RAMAN, A. 2005. Gall-inducing insects - Nature's most sophisticated herbivores. Basic Appl. Ecol. 6(5):407-411. http://dx.doi.org/10.1016/j.baae.2005.07.001

STONE, G.N. \& SCHÖNROGGE, K. 2003. The adaptative significance of insect gall morphology. Trends Ecol. Evol. 18(10):512-522. http://dx.doi. org/10.1016/S0169-5347(03)00247-7

URSO-GUIMARÃES, M.V.; SCARELI- SANTOS C. \& BONIFÁCIOSILVA A.C.. 2003. Occurrence and characterization of entomogen galls in plants from natural vegetation areas in Delfinópolis, MG. Brazil. Braz. J. Biol. 63(4):705-715. PMid:15029382. http://dx.doi.org/10.1590/ S1519-69842003000400018

URSO-GUIMARÃES, M.V. \& SCARELI-SANTOS, C. 2006. Galls and gall makers in plants from the pé-de-gigante cerrado reserve, Santa Rita do Passa Quatro, SP, Brazil. Braz. J. Biol. 66(1): 357-369.

VELDTMAN, R. \& McGEOCH, M.A. 2003. Gall-forming insect species richness along a non-scleromorphic vegetation rainfall gradient in South Africa: the importance of plant community composition. Austral Ecol. 28(1):1-13. http://dx.doi.org/10.1046/j.1442-9993.2003.01234.x 